an early prognostic marker for later development of the metabolic syndrome. Lack of difference in obestatin concentrations between LGA and AGA groups could possibly suggest that obestatin may not be directly involved in the regulation of fetal adiposity and insulin sensitivity.

\section{EFFECT OF FASTING ON METABOLISM IN TRANSGENIC MICE WITH RESPIRATORY CHAIN COMPLEX III DEFICIENCY}

doi:10.1136/archdischild-2012-302724.0221

${ }^{1} \mathrm{~N}$ Ljubas, ${ }^{1} \mathrm{E}$ Hansson, ${ }^{1} \mathrm{H}$ Kotarsky, ${ }^{1,2} \mathrm{~V}$ Fellman. ${ }^{1}$ Department of Pediatrics, University of Lund, Lund, Sweden; 'Department of Pediatrics, University of Helsinki, Helsinki, Finland

Background and aim To generate ATP production, mitochondria host crucial metabolic pathways that interact continuously. Therefore, pathological interruptions in one process might disturb entire cell metabolism. To investigate a neonatal mitochondrial disorder (GRACILE syndrome), we developed a mouse model with c.232A $>$ G mutation in Bcs 11 , resulting in a lethal complex III (CIII) deficiency in homozygotes. Our aim was to analyze how CIII deficiency affects metabolic pathways by pressing the mechanisms with fasting.

Methods Homozygous $\left(B \operatorname{cs} 1 l^{G / G}\right)$ and wild type $\left(B \operatorname{cs} 11^{A / A}\right)$ mice were assessed before and after 4-hour fasting with blood glucose, lactate and ketones, and sacrificed. Liver tissue was obtained for histology (H\&E, PAS staining for glycogen and ORO-staining for fat) and ATP measurement.

Results Before fasting, $B \operatorname{cs} 1 /^{G / G}$ had lower glucose $(4.3 \pm 1.3$ vs. $6.6 \pm 1.2, p<0.01)$ and higher ketone $(0.6 \pm 0.3$ vs. $0.3 \pm 0.1, p<0.01)$ levels, but similar lactate values $(4.0 \pm 2.2$ vs. $3.7 \pm 1.4 \mathrm{p}=0.8)$. Glycogen depletion and microvesicular steatosis present in $B c s 1 l^{G / G}$ hepatocytes increased after fasting. After fasting, $B c s 1^{A / A}$ remained euglycemic with increased ketone body production, whereas in $B \operatorname{cs} 1 /^{G / G}$ mice glucose, ketone and lactate were lower. ATP production of $B \operatorname{cs} 1 l^{G / G}$ mice was lower than that of $B \operatorname{cs} 1 l^{A / A}(58 \% \pm 24 \%)$.

Conclusion $B \operatorname{cs}_{1 / G / G}$ mice switched their metabolism to $\beta$-oxidation before fasting and failed to build up compensatory metabolic mechanisms to fasting, resulting in low ATP production. These results elucidate mechanisms explaining the deterioration in $B \operatorname{cs} / /^{G / G}$ mice. The methods used can be implemented as outcome measures in intervention studies aiming at stimulating mitochondrial biogenesis and metabolism in the mouse model.

\section{BRAIN VOLUMETRY IN INFANTS WITH CONGENITAL HEART DISEASE: PRE- AND POST-SURGERY ASSESSMENTS COMPARED TO HEALTHY CONTROLS}

doi:10.1136/archdischild-2012-302724.0222 ${ }^{6} \mathrm{~V}$ Bernet, ${ }^{5} \mathrm{~W}$ Knirsch, ${ }^{2} \mathrm{~B}$ Latal, Heart and Brain Research Group. 'Developmental Pediatrics, Kantonsspital, Winterthur, ${ }^{2}$ Child Development Center, University Children's Hospital, Zurich, Switzerland; ${ }^{3}$ Child Neurology, University Children's Hospital, Mainz, Germany; ${ }^{4}$ Neonatology, University Hospital Zurich; ${ }^{5}$ Pediatric Cardiology; ${ }^{6}$ Neonatology and Intensive Care, University Children's Hospital, Zurich, Switzerland

Background and aims MRI studies in neonates with congenital heart disease (CHD) have demonstrated delayed brain maturation and mostly focal brain injury. To better define the distribution of cerebral injuries and regional brain growth in neonates with CHD, we compared volumetric measures from pre- and postoperative MRI of patients to healthy neonates.

Methods Cerebral MRIs of 32 term-born CHD patients, scanned before and after heart surgery (mean age: 6.8 days and 26.8 days, respectively), were manually segmented to measure volumes of total, grey and white matter and of selected brain regions. Results were compared with MRIs of 17 healthy term born neonates (mean age: 23.5 days).
Results Between pre-and postoperative MRI, patients showed significant brain growth, especially in the cortical grey matter $(0.25 \%$ / day), cerebellum $(0.20 \% /$ day $)$, and deep gray matter structures $(0.10-0.15 \% /$ day, all $\mathrm{p}<0.004)$. Volume increase of the white matter was $0.05-0.06 \%$ /day (left/right; $\mathrm{p}=0.017 / 0.003$ ); increase of total brain volume was $0.14 \%$ /day $(\mathrm{p}<0.001)$.

Compared to healthy controls, the size of all brain structures (except ventricles and right amygdala) was significantly reduced postoperatively. Largest differences were found in deep gray matter structures $(13.8-16.8 \%, p=0.05-<0.001)$, cortical grey $(12.1 \%$, $\mathrm{p}=0.01)$ and white matter $(11.8 \%, \mathrm{p}<0.001)$. Total brain volumes were reduced by $11.3 \%(p<0.001)$.

Conclusions In neonates with $\mathrm{CHD}$, significant differences of white and deep grey matter volumes were found postoperatively. Brain growth was high, with notable regional differences. Our results contribute to the knowledge on the timing of cerebral injury in neonates with CHD.

\section{CLINICAL UTILITY OF AN AUTOMATED NEONATAL SEIZURE DETECTION ALGORITHM}

doi:10.1136/archdischild-2012-302724.0223

${ }^{1} \mathrm{~N}$ Stevenson, ${ }^{2} \mathrm{~S}$ Mathieson, ${ }^{3} \mathrm{~A}$ Temko, ${ }^{3} \mathrm{D}$ Dwyer, ${ }^{1} \mathrm{E}$ Low, ${ }^{3} \mathrm{G}$ Lightbody, ${ }^{2} \mathrm{~J}$ Rennie, ${ }^{3} \mathrm{~W}$ Marnane, ' $\mathrm{G}$ Boylan. ${ }^{1}$ Pediatrics and Child Health, University College Cork, Cork, Ireland; 'University College London Hospital, London, UK; ${ }^{3}$ Electrical Engineering, University College Cork, Cork, Ireland

Background/Aims EEG is the gold standard for the identification of neonatal seizures as the vast majority of electrographic seizures do not have a clinical correlate. Both under and over diagnosis of seizures is common in the neonatal intensive care unit (NICU). Computer assisted methods of interpreting the EEG have the potential to improve the accuracy of seizure detection. The aim of this study was to determine the clinical utility of our current neonatal seizure detection algorithm (NSDA).

Methods Multi-channel video-EEG recordings of 70 term neonates admitted to the NICU were analysed: 35 babies with seizure (mixed aetiologies) and 35 babies without seizure. The EEGs were annotated by an experienced neurophysiologist. The performance of the NSDA was assessed using time and event based metrics. An additional, clinically relevant, performance metric (based on the number of neonates correctly administered an anti-epileptic drug (AED) as early as possible after electrographic seizure onset) was calculated.

Results The sensitivity and specificity of the NSDA were $83 \%$ and $97 \%$ respectively when comparing to the experts annotation. The seizure detection rate and false alarm rate were $80 \%$ and $0.7 / \mathrm{hr}$ respectively. Thirty-four percent of neonates with seizures received an AED within the defined optimal timeframe, while $20 \%$ of neonates without seizure received an AED. These results were improved to $71 \%$ and $11 \%$, respectively, by supplementing decision making with the output of the NSDA.

Conclusion Current NSDA performance, while not perfect, would greatly improve the efficacy of seizure detection and optimal AED administration in the NICU.

\section{AEEG AND NIRS DURING TRANSITION AFTER BIRTH}

doi:10.1136/archdischild-2012-302724.0224

${ }^{1} \mathrm{G}$ Pichler, ${ }^{\prime} \mathrm{A}$ Avian, ${ }^{1} \mathrm{C}$ Binder, ${ }^{1} \mathrm{H}$ Zotter, ${ }^{2} \mathrm{GM}$ Schmölzer, ${ }^{1} \mathrm{~N}$ Morris, 'W Müller, ${ }^{1} \mathrm{~B}$ Urlesberger. 'Medical University of Graz, Graz, Austria; ${ }^{2}$ Royal Alexandra Hospital, Edmonton, $A B$, Canada

Background and aims Easily applicable non-invasive devices to monitor cerebral activity and oxygenation continuously during neonatal transition and resuscitation are lacking. We aimed to identify 